\title{
RELAÇÃO ENTRE AS CRENÇAS DE AUTO-EFICÁCIA E O APOIO SOCIAL NA TRANSIÇÃO PARA O TRABALHO EM ESTUDANTES FINALISTAS DO ENSINO SUPERIOR
}

\author{
Sara Samssudin ${ }^{1}$ \\ Alexandra Barros ${ }^{2}$
}

\begin{abstract}
Resumo: Com base na teoria sócio-cognitiva de Bandura (1995), a presente investigação teve como objectivo estudar a relação entre as crenças de auto-eficácia na transição para o trabalho e o apoio social percebido em estudantes finalistas do ensino superior. A amostra foi constituída por 221 participantes. Os instrumentos utilizados foram a Escala de Auto-eficácia na Transição para o Trabalho (Vieira \& Coimbra, 2005) e a Escala de Apoio Social (Vaux et al., 1986; versão adaptada por Antunes \& Fontaine, 1994/5). Os resultados indicaram uma relação positiva e significativa entre as crenças de auto-eficácia e o apoio social percebido no que diz respeito ao apoio social percebido por parte de amigos e de outros em geral.
\end{abstract}

Palavras-chave: Transição, Trabalho, Ensino Superior, Auto-eficácia, Apoio Social

Relation between the beliefs of self-efficacy and the social support on the transition to work on graduate students in higher education (Abstract): Based on the socio-cognitive theory of Bandura (1995), the aim of the present research was to study the relation between self-efficacy beliefs in the transition to work and perceived social support of college students. The sample was constituted by 221 participants. The instruments used were the Scale of Self-efficacy in the Transition to Work (Vieira \& Coimbra, 2005) and the Social Support Scale (Vaux et al., 1986; adapted version by Antunes \& Fontaine, 1994/5). The results revealed a positive and significant relation between self-efficacy beliefs and perceived social support, especially regarding perceived social support from friends and general others.

Keywords: Transition, Work, Higher Education, Self-efficacy, Social Support

\footnotetext{
1 sara.smdn@gmail.com

2 afbarros@fp.ul.pt
} 
As evidências reunidas nas últimas décadas têm demonstrado que a transição do ensino superior para o mercado de trabalho é um processo particularmente desafiante, exigindo que os jovens mobilizem os seus recursos, no confronto com múltiplas e complexas tarefas (nos domínios académico, social, relacional, vocacional e emocional das suas vidas), implementem novas estratégias e resolvam adequadamente as tarefas desenvolvimentistas para que possam obter sucesso na vida profissional (Cohen-Scali, 2003). Este processo toma hoje uma importância acrescida já que ao longo dos últimos anos tem existido uma diminuição da percentagem de diplomados que consegue emprego logo após terminar o curso (Alves, 2005). Ou seja, o número de licenciados continua a aumentar e o mercado de trabalho continua a estreitar-se, para esta população (CNAVES, 2004).

Nos estudos da área da Psicologia que tomam como ponto de partida as teorias de desenvolvimento vocacional, considera-se que a transição para o trabalho é uma componente desse desenvolvimento. Esta componente corresponde a um processo dilatado no tempo, uma vez que se inicia antes da conclusão do percurso formativo e, continua mesmo após o início da actividade profissional (Lent, Hackett \& Brown, 1999; Reitzle, Vondracek \& Silbereisen, 1998; Savickas, 1999; Super, 1990; Super, Savickas \& Super, 1996; Vieira, Maia \& Coimbra, 2007). A literatura vocacional salienta o carácter activo e participativo dos indivíduos e considera o seu potencial de influência nesta transição (Lent \& Worthington, 1999; Savickas, 1999), enfatizando como o jovem adulto pode optimizar o seu papel num processo que é também influenciado tanto por factores sociais como por factores económicos (Blustein, 1999).

Apesar de se considerar a influência de vários factores no processo de transição para o trabalho, o presente estudo vai abordar, especificamente, a relação entre dois antecedentes que parecem, de acordo com a literatura, estar integrados neste processo, nomeadamente, a auto-eficácia e o apoio social.

A auto-eficácia consiste " $n a$ crença do indivíduo sobre a sua capacidade para organizar e executar as acções requeridas para lidar com situações futuras" (Bandura, 1995, p. 2). Estas crenças de auto-eficácia influenciam, por sua vez, as escolhas realizadas, o esforço empreendido, a persistência perante o confronto com obstáculos, a qualidade do desempenho e, também, a forma como as pessoas se sentem (Bandura, 1997). Deste modo, as crenças de auto-eficácia são encaradas como um factor psicológico envolvido no processo de transição, uma vez que poderão ter uma grande influência no optimismo do jovem (Betz, 2004).

O apoio social pode definir-se como "a existência ou disponibilidade de pessoas em quem se pode confiar, pessoas que nos mostram que se preocupam connosco, nos valorizam e gostam de nós" (Sarason, Levine, Basham 
\& Sarason, 1983, p. 127). A literatura acerca do apoio social defende que este parece facilitar a adaptação do sujeito às mudanças (Antunes \& Fontaine, 1994/95), as transições em vários níveis escolares e o bem-estar em contexto académico (Balk, Tyson-Rawson \& Colletti-Wetzel, 1993;Wang \& Castañeda-Sound, 2008; Zaleski, Levey-Thors \& Schiaffino, 1998).

Analisando os conceitos de Bandura (1997), acerca das quatro fontes de informação que criam e consolidam as crenças de auto-eficácia, pode prever-se uma relação entre as crenças de auto-eficácia e o apoio social. A observação ou o acesso a relatos de histórias bem sucedidas de pessoas com as quais se estabelecem relações sociais ou pessoas que fornecem apoio social, bem como as influências directas (i.e., persuasão social) por parte dessas pessoas podem fomentar ou fortalecer as crenças de auto-eficácia.

Além disso, se atendermos, mais especificamente, às necessidades de apoio durante a transição, identificadas por Goodman e Hoppin (1990, citados por Schlossberg et al., 1995), ligadas a um sentimento positivo acerca de si próprio (proporcionado pelas pessoas que gostam do indivíduo e que acreditam nas suas capacidades) e ao encorajamento (fornecido pelas pessoas que esperam que o indivíduo seja bem sucedido), estamos perante uma importante fonte de auto-eficácia, nomeadamente, a persuasão verbal e as influências sociais que informam o indivíduo acerca da percepção que os outros têm das suas capacidades (Bandura, 1997).

Neste sentido, Lent et al. (2001) salientaram a relação, mediada pela auto-eficácia, entre o apoio e as barreiras sociais e as escolhas vocacionais dos estudantes universitários. Segundo Vieira (2008), o apoio social poderá funcionar como uma fonte de auto-eficácia, na medida em que é provável que, a partir de um contexto percebido como apoiante, estejam presentes verbalizações que promovam a confiança do indivíduo nas suas próprias capacidades. Esta autora propõe ainda que o apoio social reflecte, de forma indirecta, experiências de aprendizagem importantes para a capacidade de enfrentar os desafios colocados na transição para o mercado de trabalho.

Em síntese, a literatura sócio-cognitiva concebe o apoio social como fonte de auto-eficácia (por exemplo, Bandura, 1997;Lent et al., 2001; Vieira, 2008), parecendo existir várias formas através das quais o apoio social se relaciona com as crenças de auto-eficácia.

Por outro lado, a conceptualização do apoio social refere-se a funções desempenhadas por grupos (como, por exemplo, familiares, amigos e professores) para o indivíduo, em determinadas situações da sua vida. Os vários grupos que constituem as fontes de apoio social têm impacto diferente consoante a faixa etária, o estádio de desenvolvimento e as necessidades do indivíduo (Antunes \& Fontaine, 2005; Argyle, 1992; Srebnik \& Cauce, 1988; Walen \& Lachman, 2000). Pretende-se, neste sentido, compreender quais as fontes de apoio mais valorizadas pelos jovens adultos e que têm, 
eventualmente, maior relação com a sua auto-eficácia durante a transição para o mercado de trabalho.

Relativamente à importância das diferentes fontes de apoio social nesta relação, Srebnik e Cauce (1988) sugerem que, relativamente a questões relacionadas com a escola ou o trabalho, os indivíduos tendem a considerar a fonte de apoio formal (como por exemplo, os professores) como sendo mais relevante, enquanto a família e os amigos são mais importantes no apoio emocional. Por outro lado, Vieira (2008), relacionando o apoio social com a auto-eficácia na transição para o trabalho, propõe que a família é a fonte de apoio onde esta relação é mais evidente.

Por último, tendo em conta o aumento do número de jovens que frequentam o ensino superior, bem como as crescentes taxas de desemprego dos indivíduos jovens com formação superior, considerou-se que esta temática deve ser alvo de interesse dos investigadores e que devem ser realizadas intervenções no sentido de facilitar este processo (Alves, 2005; CNAVES, 2004). Desta forma, o presente estudo tem como objectivo central analisar a relação entre o apoio social (da família, dos amigos e dos professores) e as crenças de auto-eficácia no processo de transição para o trabalho em estudantes do ensino superior. Procura-se, assim, dar resposta aos seguintes problemas de investigação:

1) $\mathrm{O}$ apoio social percebido relaciona-se positivamente com a auto-eficácia na transição para o mercado de trabalho?;

2) Qual ou quais a(s) fonte(s) de apoio social em que esta relação é mais evidente?;

3) A auto-eficácia pode ser explicada a partir das diferentes fontes de apoio social (família, amigos, professores e/ou outros em geral)?

\section{Metodologia}

\subsection{Participantes}

Admitindo que o processo de transição começa antes do término do percurso formativo (Lent et al., 1999; Reitzle et al., 1998; Savickas, 1999; Super, 1990; Super et al., 1996; Vieira, et al., 2007), os estudantes finalistas encontram-se a vivenciar este processo de transição. Neste sentido, a metodologia adoptada teve em atenção o facto de todos os participantes a quem foram administrados os questionários serem finalistas do ensino superior (de uma Licenciatura ou de um Mestrado Integrado) e terem uma idade máxima de 25 anos. 
Os questionários abaixo caracterizados foram então administrados a 221 participantes. Os dados recolhidos integraram alunos de vários cursos diferentes, na tentativa de representar vários perfis motivacionais. Todos os participantes estudavam na área da Grande Lisboa, sendo, particularmente, alunos finalistas da Licenciatura ( $3^{\circ}$ ano) dos cursos de Comunicação e Cultura e de Estudos Artísticos (variante de Artes do espectáculo) na Faculdade de Letras da Universidade de Lisboa (UL), de Ciências da Educação na Faculdade de Psicologia e de Ciências da Educação da UL, de Biologia na Faculdade de Ciências da UL e Economia no Instituto Superior de Ciências do Trabalho e da Empresa (ISCTE); alunos finalistas da Licenciatura ( $4^{\circ}$ ano) de Ortoprotesia da Escola Superior de Tecnologias de Saúde de Lisboa e Informática e Gestão de Empresas no ISCTE; e alunos finalistas de Mestrado Integrado ( $5^{\circ}$ ano) de Engenharia Civil na Faculdade de Ciências e Tecnologias da Universidade Nova de Lisboa. Esta amostra foi constituída por conveniência, após contacto com professores e/ou coordenadores de gabinetes de apoio psico-pedagógico das faculdades e ida às turmas para aplicação dos questionários.

A amostra foi composta por 90 participantes do sexo masculino (correspondendo a $40,7 \%$ da amostra total) e 131 participantes do sexo feminino (correspondendo a 59,3\% da amostra total) com idades compreendidas entre os 19 e os 25 anos $(M=21.52$; $\mathrm{SD}=1.40)$.

\subsection{Instrumentos}

A escala de Auto-Eficácia na Transição para o Trabalho (AETT; Vieira \& Coimbra, 2005) é um instrumento de auto-relato que tem como objectivo avaliar a auto-eficácia face à transição do ensino superior para o trabalho, ou seja, a medida em que o sujeito se sente confiante na sua capacidade para realizar tarefas relacionadas com a procura de emprego, com o início da actividade profissional e respectiva adaptação ao trabalho. Este instrumento é constituído por 28 itens agrupados em três subescalas: a subescala de Auto-eficácia na Adaptação ao Trabalho constituída por 12 itens (e.g. "Resolver problemas inesperados no meu trabalho", $\alpha=.95$ ), a subescala de Auto-eficácia na Regulação Emocional constituída por 9 itens (e.g. "Após uma recusa de emprego, não me deixar invadir pelo desânimo", $\alpha=.93$ ) e a Auto-eficácia na Procura de Emprego constituída por 7 itens (e.g. "Responder a anúncios de emprego do jornal" $\alpha=0,84$ ). As opções de resposta a estes itens organizam-se numa escala de Likert 1 a 6, em que 1 significa "Nada Confiante" e 6 "Totalmente confiante". De um modo geral, os resultados dos estudos da AETT mostraram uma estrutura tridimensional e indicaram que a escala pode ser utilizada, com relativa confiança, para avaliar os níveis de auto-eficácia face à adaptação ao trabalho, à regulação emocional e à procu- 
ra de emprego (dados mais detalhados em Samssudin, 2009; Vieira \& Coimbra, 2005).

A escala de Apoio Social (SSA; Social Support Appraisals; Vaux et al, 1986) utilizada neste estudo, adaptada para a população portuguesa por Antunes \& Fontaine $(1994 / 95,2005)$ consiste num instrumento de auto-relato constituído por 30 itens que avaliam o apoio social percebido. A construção desta escala baseou-se na perspectiva de apoio social como a crença de que se é amado, respeitado, estimado e afiliado a grupos. Esta crença é uma percepção subjectiva, fornecida pela ocorrência de interacções confortantes e apoiantes, relacionadas com o tamanho real das redes de apoio, a sua composição e a proximidade das relações (Vaux, 1988).

$\mathrm{Na}$ sua forma final, a versão portuguesa da escala é constituída por 4 subescalas: SSA-am (percepção de apoio social por parte de amigos, constituída por 7 itens, e.g. "Os meus amigos respeitam-me", $\alpha=.88$ ), SSA-fam (percepção de apoio social por parte da família, constituída por 8 itens, e.g. "A minha família preocupa-se bastante comigo", $\alpha=.90)$ SSA-ger (percepção de apoio social por parte dos outros em geral, constituída por 8 itens, e.g. "As pessoas, de um modo geral, gostam de mim", $\alpha=.84$ ) e SSA-prof (percepção de apoio social por parte dos professores, constituída por 7 itens, e.g. "Eu sou bastante apreciado pelos meus professores", $\alpha=.90)$. São 6 as opções de resposta aos itens desta escala, nomeadamente, "Concordo totalmente", "Concordo bastante", "Concordo um pouco", "Discordo um pouco", "Discordo bastante" e "Discordo totalmente".

Através da análise factorial exploratória encontraram-se quatro factores que correspondem às quatro subescalas acima referidas que explicam 61\%da variância.

Assim, os resultados dos estudos da escala de Apoio Social mostraram uma estrutura multidimensional que permite distinguir a percepção de apoio social de três redes potenciais - a família, os amigos e os professores (para maior detalhe consultar Antunes \& Fontaine, 1994/95; Samssudin, 2009).

\subsection{Procedimentos}

As aplicações foram efectuadas nos meses de Fevereiro e Março, correspondendo a períodos anteriores ou posteriores à época de exames, tendo-se adaptado estes momentos de aplicação ao calendário lectivo de cada faculdade. Estas aplicações realizaram-se após o contacto com o Gabinete de Apoio ao Aluno da respectiva faculdade e o envio de uma carta ao Conselho Executivo de mesma, ou a explicação do estudo aos docentes. Decorreram, em períodos de aulas estipulados pelos docentes das cadeiras, de modo a interferir o mínimo possível com as actividades planeadas. O tempo de aplicação foi de cerca de 20 minutos. 


\section{Resultados}

Para estudar os problemas de investigação 1 e 2, realizou-se a correlação entre as subescalas e os totais da escala de Auto-eficácia na Transição para o Trabalho e a escala de Apoio Social.A Tabela 1 apresenta as correlações entre as subescalas dos dois instrumentos utilizados neste estudo.

Os valores $r$ das correlações de Pearson apresentam valores que oscilam entre .05 e .39. A análise das correlações entre estas duas escalas permite verificar que todas as subescalas e totais se correlacionam positiva e significativamente entre si, exceptuando a Auto-eficácia na Procura de Emprego e o Apoio Social da Família.

Tabela 1 - Matriz de correlações entre a Auto-eficácia na Transição para o Trabalho e o Apoio Social Percebido ( $\mathrm{N}=221$ )

\begin{tabular}{lccccc}
\hline & $\begin{array}{c}\text { Apoio } \\
\text { Social da } \\
\text { Família }\end{array}$ & $\begin{array}{c}\text { Apoio } \\
\text { Social dos } \\
\text { Amigos }\end{array}$ & $\begin{array}{c}\text { Apoio Social } \\
\text { dos Profes- } \\
\text { sores }\end{array}$ & $\begin{array}{c}\text { Apoio Social } \\
\text { dos Outros } \\
\text { em Geral }\end{array}$ & $\begin{array}{c}\text { Apoio } \\
\text { Social } \\
\text { total }\end{array}$ \\
\hline $\begin{array}{l}\text { AE na Adaptação } \\
\text { ao Trabalho }\end{array}$ & $.22^{* *}$ & $.31^{* *}$ & $.25^{* *}$ & $.38^{* *}$ & $.36^{* *}$ \\
AE na Regulação & $.25^{* *}$ & $.33^{* *}$ & $.24^{* *}$ & $.37^{* *}$ & $.37^{* *}$ \\
$\begin{array}{l}\text { Emocional } \\
\text { AE na Procura de }\end{array}$ & .05 & $0,19^{* *}$ & $.15^{*}$ & $.16^{*}$ & $.17^{*}$ \\
$\begin{array}{l}\text { Emprego } \\
\text { AE na Transição } \\
\text { para o Trabalho }\end{array}$ & $.22^{* *}$ & $.35^{* *}$ & $.27^{* *}$ & $.39^{* *}$ & $.38^{* *}$ \\
total
\end{tabular}

*significativo a $\mathrm{p}<0,05 ; * *$ significativo a $\mathrm{p}<0,01$

A correlação moderada entre o resultado total da Auto-eficácia na Transição para o Trabalho e o Apoio Social total indica que existe uma relação importante entre estas duas variáveis, respondendo afirmativamente ao problema de investigação $\mathrm{n}^{\circ} 1$.

Os resultados da escala total de Auto-eficácia na Transição para o Trabalho têm uma correlação moderadamente elevada com o Apoio Social dos Outros em Geral, seguindo-se o Apoio Social dos Amigos, o Apoio Social dos Professores e, por último, o Apoio Social da Família. Em relação ao problema de investigação $\mathrm{n}^{\circ} 2$, pode considerar-se que o apoio social percebido por parte dos outros, de uma forma geral, tem uma relação importante, quer com o total, quer com as dimensões específicas das crenças de auto-eficácia analisadas. Por outro lado, esta variável parece também estar rela- 
cionada com a fonte de apoio por parte dos amigos, bem como dos professores e da família.

$\mathrm{Na}$ análise das subescalas, é também possível verificar que o Apoio Social dos Outros em Geral apresenta as correlações mais expressivas com a Auto-eficácia na Adaptação ao Trabalho e a Auto-eficácia na Regulação Emocional, embora o Apoio Social dos Amigos também apresente correlações positivas e significativas com essa escala. A Auto-eficácia na Procura de Emprego apresenta correlações positivas e significativas com o Apoio Social dos Amigos, com o Apoio Social dos Professores e com o Apoio Social dos Outros em Geral, mas relativamente mais baixas do que as outras subescalas.

Por outro lado, tanto a Auto-Eficácia na Adaptação ao Trabalho como a Auto-eficácia na Regulação Emocional apresentam correlações significativas positivas com o Apoio Social, enquanto a Auto-eficácia na Procura de Emprego apresenta uma correlação menor, mas ainda significativa.

Por último, para analisar a relação entre a auto-eficácia na transição para o trabalho total e as diferentes fontes de apoio social (problema de investigação $\mathrm{n}^{\circ} 3$ ), utilizou-se uma regressão linear múltipla. A Tabela 2 apresenta os resultados obtidos. Verifica-se que o conjunto das diferentes fontes de apoio social explica apenas $17 \%$ da variância $\left(\mathrm{R}^{2}=0,17\right)$ na Auto-eficácia na Transição para o Trabalho (AETT). O apoio social dado pelos outros em geral $(\mathrm{p}<0,01)$ é a única fonte que permite predizer a AETT. As outras variáveis não estão positivamente associadas à AETT e não são significativas para o modelo.

Tabela 2: Regressão linear múltipla: Auto-eficácia na Transição para o Trabalho e fonts de Apoio Social $(\mathrm{N}=221)$

\begin{tabular}{rcccc}
\hline & $\mathrm{B}$ & $\mathrm{Beta}$ & $\mathrm{t}$ & $\mathrm{p}$ \\
\hline Apoio Social Família & $-0,17$ & -.05 & $-0,58$ & .57 \\
Apoio Social Amigos & 0,61 & .13 & 1,41 & .16 \\
Apoio Social Professores & 0,30 & .11 & 1,48 & .14 \\
Apoio Social Outros em Geral & 1,04 & .26 & 2,54 & $.01^{* *}$ \\
\hline$* *$
\end{tabular}

**significativo a $\mathrm{p}<0,01$

\section{Conclusão}

O presente estudo pretendeu analisar a relação entre o apoio social percebido e as crenças de auto-eficácia durante o processo de transição do ensino superior para o trabalho. Procurou-se igualmente perceber qual ou 
quais as fontes de apoio social (família, amigos, professores ou os outros em geral) que apresentam uma maior relação com as crenças de auto-eficácia na adaptação ao trabalho, na procura de emprego e na regulação emocional durante esta transição. Por último, pretendeu perceber-se de que forma as diferentes fontes de apoio social permitem prever a auto-eficácia total.

A análise das correlações entre as escalas permitiu concluir que existe uma relação significativa positiva e elevada entre o apoio social e a auto-eficácia na transição para o trabalho, tal como já tinha sido referido em Vieira (2008). Estes resultados mostram que estas variáveis actuam em conjunto durante esta transição.

A auto-eficácia na transição para o trabalho parece ter uma relação mais evidente com o apoio social dos outros em geral, permitindo concluir que, de uma forma geral, a percepção de apoio por parte de outros significativos tem importantes relações com as crenças de auto-eficácia nesta transição. Contudo, a natureza dos itens desta subescala não deixa claro qual a fonte em que os sujeitos se baseiam para responder. De entre a família, os professores e os amigos, os resultados indicam uma maior relação entre a auto-eficácia total e o apoio social percebido por parte dos amigos, seguindo-se os professores e, por último, os familiares. Tendo em conta o estudo de Strebnik e Cauce (1988) relativamente à importância de fontes de apoio formal (como, por exemplo, os professores) em questões relacionadas com a escola ou o trabalho, podia explorar-se, numa futura investigação, se os amigos que os jovens consideram são os colegas de curso, o que indicaria uma relevância dos indivíduos que também passam pela mesma transição.

É interessante notar que a Auto-eficácia na Regulação Emocional é a que parece estar mais relacionada com o Apoio Social total, seguindo-se a Auto-eficácia na Adaptação ao Trabalho. A Auto-eficácia na Procura de Emprego apresentou relações menos evidentes com o Apoio Social, podendo colocar-se a hipótese de que as condições do mercado de trabalho, no momento actual, podem ter reduzido a relevância do apoio social. Em todo o caso, os resultados sugerem que os outros significativos são importantes para a eficácia percebida relativamente a factores mais emocionais nesta transição.

De uma forma geral, estes resultados sustentam a previsão inicial relativamente à relação entre o apoio social e a auto-eficácia na transição para o trabalho, o que está de acordo com os resultados encontrados por Vieira (2008). Contudo, nos resultados encontrados por estas autoras a família foi a fonte cujo apoio mais se relacionou com as crenças de auto-eficácia na transição para o trabalho, enquanto os resultados deste estudo sugerem que são os amigos a fonte cujo apoio se relaciona mais com as crenças de auto-eficácia nesta transição. A diferença entre os resultados encontrados pode dever-se à extensão das idades da amostra, uma vez que, no estudo de Vieira (2008) a amostra era constituída por participantes cujo intervalo de idades 
era relativamente superior às idades dos participantes deste estudo. De acordo com as conclusões de Levitt, Weber e Guacci (1993), os jovens adultos percebem um maior apoio por parte dos amigos mas, conforme a idade vai aumentando, a percepção deste apoio vai-se reduzindo, passando a família a ser a fonte de apoio mais importante.

Por último, foi possível perceber que a Auto-eficácia total pode ser parcialmente explicada pelo Apoio Social de Outros em Geral, o que mais uma vez indica que, de uma forma geral, a percepção de apoio por parte de elementos significativos influencia as crenças de auto-eficácia durante esta transição.

As conclusões apresentadas permitem formular algumas propostas e conceber implicações práticas neste âmbito. Tomando como ponto de partida a perspectiva de que a transição para o trabalho corresponde a um processo alargado no tempo, que começa antes da conclusão do percurso formativo e continua após o início da actividade profissional (Lent et al., 1999; Reitzle et al., 1998; Savickas, 1999; Super, 1990; Super et al., 1996; Vieira et al., 2007), seria interessante prosseguir esta linha de investigação. Poder-se-ia, neste sentido, estudar a relação entre estas duas variáveis através de um estudo longitudinal, que contemplasse o momento após o término do curso e o momento de procura de emprego e/ou a adaptação ao emprego (i.e., alguns meses após a conclusão do percurso formativo, após o confronto com a realidade). Seria, por outro lado, interessante explorar a direcção da relação encontrada entre a auto-eficácia na transição para o trabalho e o apoio social percebido.

De acordo com alguns autores (por exemplo, Cramer, 2006; Heller, Swindle \& Dusenbury, 1986) que referem a importância do apoio social por parte do parceiro amoroso na percepção total de apoio social e na relação com outras variáveis pessoais, poder-se-ia também estudar a relação entre esta fonte de apoio e a auto-eficácia nesta transição.

Finalmente, este estudo apresenta indícios importantes para posteriores intervenções ao nível do ensino superior. Uma vez que a auto-eficácia parece ser um importante factor para a transição bem sucedida (Betz, 2004), seria interessante que as instituições deste nível de ensino fomentassem as percepções de competência através, por exemplo, de Workshops, de acções para a promoção de competências de empregabilidade, de actividades de Role-playing e de experiências de trabalho ao longo da formação académica. Por outro lado, uma vez que o apoio social, especificamente o apoio social dos amigos, parece ter uma relação positiva com a auto-eficácia, estas instituições podiam estimular a percepção de apoio social por parte dos amigos, o que podia ser feito através, por exemplo, da criação de grupos de pares, onde se desenvolvesse um sentimento de identidade e pertença a um grupo, de grupos de "mentorado", grupos de reflexão conjunta sobre determinadas 
temáticas, partilhas de experiências, ou mesmo partilha de receios e preparação para o momento de transição para o trabalho. Estas dinâmicas podem ser importantes para fornecer ao jovem competências que lhe permitam ser bem sucedido na transição para o trabalho, tendo em conta que, nos períodos de transição,se enfrentam complexos e constantes ajustamentos ao meio. Neste confronto, não só as oportunidades e os factores sócio-económicos do meio envolvente, mas também as competências e as crenças, influenciam o percurso profissional do indivíduo. Neste sentido, é fulcral o desenvolvimento de esforços para preparar e capacitar os jovens para um confronto adaptado e bem sucedido com a transição do ensino superior para o trabalho.

\section{Referências}

Alves, N. (2005). Trajectórias académicas e inserção profissional dos licenciados pela Universidade de Lisboa (1999-2003). Lisboa: Reitoria da Universidade de Lisboa.

Antunes, C., \& Fontaine, A. M. (1994/95). Diferenças na percepção de apoio social na adolescência: Adaptação de uma escala, o "social support appraisals" (SSA) de Vaux et al. (1980). Cadernos de Consulta Psicológica, 10/11, 115-127.

Antunes, C., \& Fontaine, A. M. (2005). Percepção de apoio social na adolescência: Análise factorial confirmatória da escala social support appraisal. Paideia, 15 (32), 355-366.

Argyle, M. (1992). Benefits produced by supportive social relationships. In H. Veiel and U. Baumann (Eds.).The meaning and measurement of social support (pp. 13-32). New York: Hemisphere Publishers.

Balk, D.E., Tyson-Rawson, K., \& Colletti-Wetzel, J. (1993). Social support as an intervention with bereave college students. Death Studies, 17, 427-450.

Bandura, A. (1995). Self-efficacy in changing societies. Cambridge, UK: Cambridge University Press.

Bandura, A. (1997). Self-efficacy: the exercise of control. New York: W.H. Freeman.

Betz, N. E. (2004). Contributions of self-efficacy theory to career counseling: A personal perspective. The Career Development Quarterly, 52 (4), 340-353.

Blustein, D.L. (1999). A match made in heaven? Career development theories and the school-to-work transition. The Career Development Quarterly, 47 (4), 348-352 .

CNAVES (Conselho Nacional de Avaliação do Ensino Superior) (2004). Parecer sobre a regulação da oferta de diplomados do ensino superior. Disponível em http://168.144.195.227/cnaves1/DOCS/Pareceres/PARECER_oferta_diplomad os.pdf .

Cohen-Scali, V. (2003). The influence of family, social, and work socialization on the construction of the professional identity of young adults. Journal of Career Development, 29 (4), 237-249. 
Cramer, D. (2006). How a supportive partner may increase relationship satisfaction. British Journal of Guidance \& Counseling, 34 (1), 117-131.

Heller, K, Swindle, R. W, \& Dusenbury, L. (1986). Component social support processes: Comments and integration. Journal of Consulting and Clinical Psychology, 54 (4),466-470.

Lent, R. B., Brown, S. D., Brenner, B., Chopra, S. B., Davis, T., Suthakaran, V., \& Talleyrand, R. (2001). The role of contextual supports and barriers in the choice of math/science educational options: A test of social cognitive hypotheses. Journal of Counseling Psychology, 48 (4), 474-483.

Lent, R. W., Hackett, G.,\& Brown, S. D. (1999). A social cognitive view of school-to-work transition. The Career Development Quarterly, 47 (4), 297-311.

Lent, R.W., \& Worthington, R.L. (1999). Apllying career development theories to the school-to-work transition process. The Career Development Quarterly, 47 (4), 291-296.

Levitt, M.J., Weber, R.A., \& Guacci, N. (1993). Convoys of social support: An intergenerational analysis. Psychology and Aging, 8 (3), 323-326.

Reitzle, M., Vondracek, F.W., \& Silbereisen, R.K. (1998). Timing of school-to-work transitions: A developmental-contextual perspective. International Journal of Behavioral Development, 22 (1), 7-28.

Samssudin, S. (2009). Relação entre as crenças de auto-eficácia e o apoio social na transição para o trabalho em estudantes finalistas do ensino superior. Dissertação de mestrado integrado em psicologia da educação e da orientação apresentada à Faculdade de Psicologia e de Ciências da Educação da Universidade de Lisboa.

Sarason, I. G., Levine, H. M., Basham, R. B., \& Sarason, B. R. (1983). Assessing social support: The social support questionnaire. Journal of Personality and Social Psychology, 44 (1), 127-139.

Savickas, M. L. (1999). The transition from school to work: A developmental perspective. The Career Development Quarterly, 47 (4), 326-336.

Schlossberg, N. K., Waters, E.B., \& Goodman, J. (1995). Counseling adults in transition. New York: Springer Publishing Company.

Srebnik, D., \& Cauce, A.M. (1988). Social support providers: A multidimensional analysis of network systems. American Psychological Association. Atlanta, Georgia.

Super, D. E. (1990). A life-span, life-space approach to career development. In D. Brown e L. Brooks (Eds.), Career Choice and Development. Applying Contemporary Theories to Practice (2nd edition, pp. 197-261). San Francisco, CA: Jossey-Bass Publishers.

Super, D. E., Savickas, M. L., \& Super, C. M. (1996). The life-span, life-space approach to careers. In D. Brown \& L. Brooks and al (Eds.), Career Choice and Development (3rd edition, pp. 121-178). San Francisco, CA: Jossey-Bass Publishers.

Vaux, A. (1988). Social Support: Theory, research and intervention. New York: Praeger. 
Vieira, D. \& Coimbra, J. L. (2005). University-to-work transition: The development of a self-efficacy scale. Conference proceedings of AIOSP International Conference 2005 - Careers in context: New challenges and tasks for guidance and counselling (p. 106). Lisboa: Universidade de Lisboa.

Vieira, D. (2008). Perspectiva sociocognitiva da transição do ensino superior para o trabalho: Influência da auto-eficácia e dos objectivos no sucesso de uma transição vocacional. Dissertação de doutoramento apresentada à Faculdade de Psicologia e de Ciências da Educação da Universidade do Porto.

Vieira, D., Maia, J., \& Coimbra, J.L. (2007). Do ensino superior para o trabalho: Análise factorial confirmatória da escola de auto-eficácia na transição para o trabalho (AETT). Avaliação Psicológica, 6 (1), 3-12.

Walen, H. R., \& Lachman, M. E. (2000). Social support and strain from partner, family, and friends: Costs and benefits for men and women in adulthood. Journal of Social \& Personal Relationships, 17 (1), 5-30.

Wang, C. D. C., \& Castañeda-Sound, C. (2008). The role of generational status, self-esteem, academic self-efficacy, and perceived social support in college students' psychological well-being. Journal of College Counseling, 11, 101-118.

Zaleski, E. H., Levey-Thors, C., \& Schiaffino, K. M. (1998). Coping mechanisms,stress, social support, and health problems in college students. AppliedDevelopmentalScience, 2 (3), 127-137. 